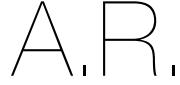

ARTIGO DE REVISÃO

${ }^{1}$ Escola Superior de Tecnologia da Saúde de Lisboa,

Av. D. João II, Lote

4.69 .01

1990-096 Lisboa, Portugal

2 Loveat,

Avenida Ventura Terra 5

$1{ }^{\circ}$ andar,

1600-780 Lisboa, Portugal

*Endereço para correspondência:

Mariana Fernandes

Escola Superior de Tecnologia

de Saúde de

Av. D. João Lisboa,

Av. D. Joăo II, Lote 4.69.01,

1990-096 Lisboa, Portugal

mariana.ferna@hotmail.com

Histórico do artigo

Recebido a 14 de outubro de 2020 Aceite a 28 de dezembro de 2020

\title{
PAPEL DO NUTRICIONISTA NUMA DIETA RESTRITA EM FODMAPs
}

\author{
THE ROLE OF A DIETITIAN IN THE LOW FODMAP DIET
}

Mariana Fernandes ${ }^{1 *}$; Mafalda Rodrigues de Almeida²; Vânia Costa ${ }^{1}$

RESUMO

A dieta restrita em FODMAPs (Fermentable Oligosaccharides, Disaccharides, Monosaccharides and Polyols) surgiu como estratégia para controlar os sintomas de doenças como a síndrome do intestino irritável (SII). Ao longo dos anos, têm sido publicados resultados positivos desta dieta. No entanto, a sua implementação não está ainda padronizada, podendo ser desafiante implementá-la com sucesso. Neste sentido, o nutricionista é um aliado importante na implementação da dieta restrita em FODMAPs e na orientação alimentar.

Assim, o objetivo desta revisão foi analisar o papel do nutricionista na implementação de uma dieta restrita em FODMAPs.

\section{PALAVRAS-CHAVE}

Dieta, FODMAPs, Nutricionista, Síndrome do Intestino Irritável

\section{ABSTRACT}

In order to control the symptoms of diseases such as irritable bowel syndrome (IBS), the low FODMAP diet (Fermentable Oligosaccharides, Disaccharides, Monosaccharides and Polyols) emerged. Over the last years, positive results of this diet have been published. However, its implementation is not yet standardized, and it can be challenging to implement it successfully. Thereby, the nutritionist is an important ally in the implementation of the low FODMAP diet and in the dietary guidance.

Thus, the objective of this review was to analyze the role of the nutritionist in implementing a low FODMAP diet.

\section{KEYWORDS}

Diet, FODMAP, Dietitian, Irritable bowel syndrome

\section{INTRODUÇÃO}

A dieta restrita em FODMAPs (Fermentable Oligosaccharides, Disaccharides, Monosaccharides and Polyols), cujo acrónimo significa, oligossacarídeos, dissacarídeos, monossacarídeos e polióis fermentáveis, tem vindo a ganhar relevo ao longo dos últimos anos, devido aos seus resultados positivos no controlo de sintomas associados à síndrome do intestino irritável (SII) (1-4). Esta abordagem nutricional teve um rápido desenvolvimento ao converter efeitos fisiológicos aparentemente díspares num conceito abrangente e bem estruturado, por meio da inovação e do trabalho árduo de uma equipa de investigadores, inicialmente na Universidade Monash e, atualmente, em todo o mundo (5).

O intuito da dieta restrita em FODMAPs é auxiliar os indivíduos, principalmente os doentes com SII, a controlar os sintomas gastrointestinais, através da identificação dos alimentos específicos que os desencadeiam (6-8).

Efeitos benéficos têm sido também relatados na sintomatologia gastrointestinal associada a patologias como a doença inflamatória intestinal, endometriose, cólica infantil, dispepsia funcional, fibromialgia, esclerodermia e síndrome da fadiga crónica. Contudo, ainda são necessários dados mais precisos para avaliar os efeitos desta abordagem nutricional nestas patologias $(2,6,9,10)$.

Todavia, a implementação da dieta restrita em FODMAPs requer conhecimentos sobre os mecanismos de atuação dos mesmos, composição dos alimentos, restrições e reintroduções alimentares adequadas, bem como manutenção da dieta a longo prazo (11-13). Por isso, é fundamental ser realizada sob a orientação de um nutricionista (14).

O nutricionista é um profissional de saúde que atua para a promoção da saúde e do bem-estar e na prevenção e tratamento da doença. Para isso, na prática clínica, realiza avaliação, diagnóstico, prescrição e intervenção alimentar e nutricional, tendo como base a melhor evidência científica (15). A avaliação nutricional consiste numa abordagem sistemática para avaliar, registar e interpretar os dados relevantes para a caracterização do estado nutricional (16). Abrange a recolha de dados da anamnese alimentar, 
medidas antropométricas, análise de parâmetros bioquímicos, sinais clínicos e história do doente (16). O diagnóstico baseia-se nos dados recolhidos na avaliação nutricional e permite definir qual será a intervenção nutricional a seguir. Não menos importante, a monitorização e avaliação através de uma reavaliação nutricional permite ajustar a intervenção às necessidades, dificuldades e objetivos do indivíduo (16). Assim, o nutricionista tem um papel preponderante na implementação da dieta restrita em FODMAPs.

\section{METODOLOGIA}

Foi efetuada uma pesquisa bibliográfica online, através da base de dados Pubmed, com enfoque em publicações dos últimos três anos, mediante a utilização das seguintes palavras e associações: Dietitian; FODMAPs; Implementation; Irritable Bowel Syndrome. A pesquisa foi realizada entre maio e setembro de 2020, tendo sido encontrados 125 artigos, dos quais foram utilizados 37 .

\section{Dieta Restrita em FODMAPs - Definição e Implementação}

Os FODMAPs são um conjunto de hidratos de carbono de cadeia curta com absorção particularmente lenta ou inexistente no intestino delgado $(1,7,17,18)$, sendo fermentados por bactérias intestinais, resultando na produção de gás $(6,14,18-22)$. Incluem-se os oligossacarídeos [galacto-oligossacarídeos (GOS), fruto-oligossacarídeos (FOS), isomalto-oligossacarídeos (IMO) e xilo-oligossacarídeos (XOS)], os dissacarídeos (lactose e lactulose), os monossacarídeos (frutose livre) e os polióis (com o sorbitol, manitol, maltitol, eritritol, xilitol e isomalte) fermentáveis $(17,20)$. Na Tabela 1 , encontram-se alguns dos principais alimentos com alto teor de FODMAPs (2, 23).

Antes da implementação da dieta é recomendável a avaliação pelo médico de família ou gastroenterologista para análise do quadro clínico e exclusão adequada de outros distúrbios gastrointestinais, bem como, se aplicável, fazer o diagnóstico clínico de SII. Posteriormente, deve ser feito um encaminhamento para um nutricionista que poderá implementar corretamente a terapêutica nutricional $(6-8,24)$.

A implementação divide-se em três etapas principais: restrição total, reintrodução controlada e manutenção da dieta adaptada à tolerância individual $(6,7,24)$.

Na primeira fase, há uma redução inicial de todos os alimentos ricos em FODMAPs (7). Porém, nenhum grupo alimentar deve ser excluído por completo, pelo que, em cada grupo de alimentos, devem trocar-se alimentos com alto teor de FODMAPs por alimentos com baixo teor (2, 6). Esta fase deve durar entre duas a seis semanas, devendo evitar-se períodos prolongados sob esta restrição, uma vez que poderá gerar carências nutricionais. Se houver alívio dos sintomas deverá passar-se à fase seguinte, caso contrário deverá prolongar-se até às seis semanas e no caso dos sintomas não melhorarem deverá procurar-se uma abordagem diferente $(25,26)$.

A segunda fase consiste na reintrodução controlada, ou seja, é feita uma introdução lenta dos alimentos ricos em $\operatorname{FODMAPs}(7,8)$. É improvável que todos os alimentos ricos em FODMAPs provoquem sintomas em todos os indivíduos, portanto a reintrodução progressiva é usada para identificar níveis de tolerância para cada alimento e subgrupo de FODMAPs (25). Inicialmente, as quantidades a testar devem ser pequenas, mas se o indivíduo se sentir bem deverá aumentá-las até uma dose normal de consumo desse alimento. $\mathrm{O}$ indivíduo deve primeiramente testar um alimento inserido num dos subgrupos individuais dos FODMAPs, podendo ser realizado o teste com o mesmo alimento durante três dias seguidos, aumentando a dose em $50 \%$ por dia. Posteriormente, deve testar os restantes subgrupos $(6,10,26)$. Entre cada nova introdução de alimentos pode ser dado um intervalo de um a três dias, porém os sintomas devem ser monitorizados todos os dias e não apenas nos dias de reintrodução $(10,19,25)$. Devem, igualmente, excluir-se os alimentos do grupo anteriormente testado, a menos que haja uma total ausência de sintomas com esses mesmos alimentos, situação em que os doentes podem optar por passar diretamente para a reintrodução seguinte (10). Depois deve testar a combinação entre os vários subgrupos dos FODMAPs (6, 25).

A terceira fase consiste na manutenção da dieta adaptada. O objetivo desta fase é reintroduzir o máximo de alimentos ricos em FODMAPs na dieta conforme tolerado na fase anterior, mantendo um bom controlo dos sintomas e evitando restrições desnecessárias $(7,25)$. Assim, após identificados os alimentos que desencadeiam os sintomas, estabelece-se um plano alimentar individual, onde se excluem esses mesmos alimentos - "dieta com baixo teor de FODMAPs modificada" $(6,25)$.

\section{ANÁLISE CRÍTICA}

O sucesso e adequação nutricional da dieta quando autoimplementada não são conhecidos (12). A dieta restrita em FODMAPs só foi avaliada quando orientada por nutricionistas, individualmente ou em sessões de grupo $(11,12,27,28)$. Todavia, a evidência sugere que a correta implementação e monitorização da dieta restrita em FODMAPs exige um seguimento rigoroso, no qual um nutricionista parece ser um elemento fundamental $(1,7,11,24)$. Mais ainda, observa-se uma adesão e manutenção da dieta duas vezes maior a longo-prazo em indivíduos seguidos por um nutricionista $(8,10,29)$.

Contudo, nem todos os nutricionistas têm experiência na prescrição da dieta restrita em FODMAPs $(11,12)$. Torna-se fulcral que o nutricionista tenha conhecimentos e até mesmo formação na área das doenças gastrointestinais e saiba implementar corretamente a dieta restrita em FODMAPs. Caso contrário, um seguimento nutricional desadequado e o fornecimento de dados imprecisos sobre a composição em FODMAPs dos alimentos podem levar a respostas insatisfatórias à dieta (11-13).

Antes da implementação da dieta, a consideração do tipo e severidade de sintomas do indivíduo, ingestão habitual, conteúdo nutricional da dieta e padrão de refeições são aspetos cruciais na avaliação do indivíduo, de maneira a ser possível seguir uma abordagem personalizada $(10,11)$.

\section{Tabela 1}

Fontes alimentares de cada subgrupo de FODMAP

\begin{tabular}{ll}
\multicolumn{1}{c}{ SUBGRUPO DE FODMAP } & \multicolumn{1}{c}{ ALIMENTOS COM ELEVADO TEOR EM FODMAPS } \\
Oligossacáridos & $\begin{array}{l}\text { Alcachofras, espargos, beterrabas, couve-de-bruxelas, brócolos, couve, erva-doce, alho, alho francês, quiabo, cebola, } \\
\text { ervilha, cebolinha, trigo, centeio, cevada, leguminosas, lentilhas, grão-de-bico, maçã, pêssego, dióspiro, melancia e } \\
\text { pistácio, e produtos processados }\end{array}$ \\
\hline Dissacáridos & Leite, iogurte, gelado, creme inglês e queijos de pasta mole \\
\hline Monossacáridos & Maçãs, cerejas, mangas, peras, melancia, espargos, alcachofras, ervilhas, mel exarope de milho com alto teor de frutose \\
\hline Polióis & $\begin{array}{l}\text { Maçãs, damascos, cerejas, peras, nectarinas, pêssegos, ameixas, ameixas secas, melancia, abacate, couve-flor, } \\
\text { cogumelos, ervilhas-tortas e os adoçantes artificiais sorbitol, manitol, maltitol, xilitol e isomalte }\end{array}$ \\
\hline
\end{tabular}


Cada indivíduo reage de forma diferente a cada subgrupo de FODMAPs, com variações tanto no tipo como na gravidade dos sintomas (11), e a tolerância pode também variar no tempo, por exemplo, em virtude do stress (6). Assim, durante a implementação da dieta, um nutricionista é essencial para auxiliar o indivíduo a testar cada subgrupo, avaliar a resposta às diferentes doses, a frequência de consumo e o efeito aditivo de múltiplos alimentos ricos em FODMAPs (6, 8, 10, 30).

Na prática, a monitorização dos sintomas dos doentes pode ser feita com recurso a ferramentas para classificação de sintomas $(10,11)$, sendo a mais comum o IBS Severity Scoring System (IBS-SSS), que mede a severidade dos sintomas e inclui questões relacionadas com a intensidade da dor e com a frequência em que ocorre, com a distensão abdominal, com os hábitos intestinais e com a qualidade de vida (10). Também pode recorrer-se à escala de Bristol, por forma a avaliar o padrão das fezes (11), e à escala visual analógica (VAS), para medir a resposta ao tratamento dos sintomas (10).

O nutricionista torna-se imprescindível para fornecer a informação necessária para o indivíduo compreender os mecanismos de atuação dos FODMAPs, conhecer os alimentos que deve excluir e perceber o efeito que a dose ingerida desempenha no surgimento dos sintomas, aspetos estes determinantes para uma correta implementação da dieta $(10,17,25,31)$. O nutricionista deverá fornecer ferramentas de apoio $(8,10)$, nomeadamente os materiais disponibilizados pela Monash University, incluindo a aplicação para smartphone com os dados atualizados sobre a composição dos alimentos em FODMAPs e alternativas com baixo teor de FODMAPs $(8,11,24,32)$. Atualmente, existe muita informação disponível em livros e em formato online, mas devido à natureza evolutiva da dieta, grande parte está desatualizada. Como tal, é de extrema importância escolher criteriosamente a fonte de informação $(8,10,11,13)$.

De facto, o nutricionista torna-se fundamental em todas as fases da dieta $(10,11)$.

Na fase restritiva, uma exclusão completa de todos os subgrupos de FODMAPs pode ser implementada (10), mas uma abordagem individualizada permite uma intervenção mais particular em determinadas situações $(8,11)$. Exemplos disso são: quando a ingestão de FODMAPs é excessiva, mas os sintomas são leves, sugerindo tolerância razoável e reduzida necessidade de restrição (8); ou quando é demonstrada tolerância à lactose, pelo que a lactose não tem de ser restringida (8, 10, 11); ou ainda quando o doente tem alívio significativo dos sintomas apenas com a remoção de alguns alimentos ricos em FODMAPs da dieta, de modo que apenas é necessária a exclusão de uma lista simplificada de alimentos ou de certos subgrupos específicos de FODMAPs (11).

De igual forma, o nutricionista torna-se determinante para adaptar a abordagem quando estão presentes restrições alimentares adicionais devido a condições clínicas concomitantes, como a diabetes, ou devido a padrões alimentares, como o vegetarianismo (10, 25, 33); ou mesmo quando se verificam aspetos individuais do doente que limitam a possibilidade de implementação da dieta, como a capacidade para entender a dieta ou para preparar e cozinhar, ou aspetos económicos $(8,11)$.

Por sua vez, na fase de reintrodução é essencial um acompanhamento dos doentes aproximadamente quatro a seis semanas após a consulta inicial para discutir o resultado das mudanças na dieta. Quando não se verificam melhorias, o acompanhamento é necessário para determinar qual o motivo do insucesso da abordagem, seja por baixa adesão à intervenção, ingestão inadvertida de FODMAPs, outras intolerâncias alimentares ou mesmo mecanismos não relacionados com a dieta (11). Quando há melhorias, a tolerância individual deve ser testada através de reintroduções alimentares (10). Contudo, não existe um método padronizado para esta reintrodução (8). Assim, é fundamental uma individualização das reintroduções de acordo com a tolerância e com os aspetos anteriormente mencionados para a fase restritiva. Idealmente, o nutricionista deverá promover reintroduções de pequenas quantidades de FOS e GOS, devido às suas propriedades prebióticas benéficas e aos efeitos que a sua ausência poderá causar na microbiota intestinal $(17,18,34,35)$.

Um dos problemas mais comuns decorrentes da restrição inicial dos FODMAPs é a fase de restrição ser tão eficaz no alívio dos sintomas que os doentes não estão dispostos a iniciar a fase de reintrodução dos alimentos $(7,29,36)$. Para auxiliar nesta situação, o nutricionista pode sugerir apoio psicológico, no sentido de melhorar a relação do indivíduo com a alimentação (7). Da mesma forma, a preocupação excessiva com os alimentos ingeridos pode contribuir para o aumento do risco de desenvolvimento de distúrbios alimentares, como a ortorexia nervosa $(8,37)$. Assim, o nutricionista deve comunicar com o doente de forma assertiva e clara, de maneira a fazê-lo compreender que a dieta restrita em FODMAPs não é uma abordagem para ser mantida inalterada por toda a vida, mas sim uma forma de atingir um equilíbrio entre uma menor restrição alimentar e o alívio dos sintomas (10-13).

Da mesma forma, na fase de manutenção, o nutricionista tem um papel preponderante no estabelecimento de uma "dieta com baixo teor de FODMAPs modificada", com exclusão dos alimentos que foram identificados como desencadeadores dos sintomas $(6,10,25)$. O nutricionista deve explicar ao indivíduo que os alimentos bem tolerados devem ser reintroduzidos na dieta e os alimentos moderadamente tolerados podem ser reintroduzidos ocasionalmente $(6,8,10,25)$. Os subgrupos de FODMAPs menos tolerados também podem ser reintroduzidos, mas a dosagem e a frequência de consumo precisam de ser individualizadas (25). A longo prazo, o nutricionista deve encorajar os doentes a testarem alguns dos alimentos anteriormente mal tolerados, por forma a reavaliar a tolerância, uma vez que esta se pode alterar com o tempo $(6,8,25)$.

Outra tarefa imprescindível do nutricionista é monitorizar o peso corporal e a ingestão alimentar durante todo o tratamento, como forma de verificar a adequação nutricional $(2,10,19)$.

O custo da implementação da dieta, devido à escolha de alimentos com baixo teor em FODMAPs, é muitas vezes apontado como uma limitação. Porém, o nutricionista pode orientar o indivíduo para escolhas adequadas e mais económicas (14). Para além disso, o acompanhamento parece ser tão eficaz quando realizado em grupo quanto quando realizado individualmente, pelo que poderá ser mais uma forma de diminuir os custos associados $(8,28)$.

\section{CONCLUSÕES}

A principal conclusão deste artigo é a existência de um papel imprescindível do nutricionista na correta implementação da dieta restrita em FODMAPs.

O princípio chave para o sucesso desta terapêutica nutricional parece ser, de facto, a educação e gestão alimentar. O papel do nutricionista é de extrema importância, até porque ainda se encontram em desenvolvimento protocolos padronizados para a reintrodução dos alimentos (dose, duração, subgrupo de FODMAPs), e a experiência do nutricionista pode ser determinante na construção e melhoria eficaz desses protocolos. Para além disso, dado que cada indivíduo responde de maneira diferente à dieta, a personalização é crucial para a implementação bem-sucedida da intervenção nutricional. Assim, o nutricionista guia a implementação da dieta, evitando restrições 
alimentares desnecessárias, promovendo uma melhor adequação nutricional, avaliando as respostas à dieta e aumentando a probabilidade de adesão e manutenção das restrições que efetivamente diminuem os sintomas do indivíduo.

Deste modo, é cada vez mais crucial nestas condições clínicas dar relevo ao trabalho em equipa entre médicos e nutricionistas, e ao encaminhamento entre áreas do saber, para maior satisfação e qualidade de vida dos doentes.

\section{REFERÊNCIAS BIBLIOGRÁFICAS}

1. Liu J, Chey WD, Haller E, Eswaran S. Low-FODMAP Diet for Irritable Bowel Syndrome: What We Know and What We Have Yet to Learn. Annu Rev Med. 2020;71(1):303-14. 2. World Gastroenterology Organisation. Dieta e intestino. J Clin Gastroenterol. 2018;34. 3. Schumann D, Klose P, Lauche R, Dobos G, Langhorst J, Cramer H. Low fermentable, oligo-, di-, mono-saccharides and polyol diet in the treatment of irritable bowel syndrome: A systematic review and meta-analysis. Nutrition. 2018;45:24-31.

4. Marsh A, Eslick EM, Eslick GD. Does a diet low in FODMAPs reduce symptoms associated with functional gastrointestinal disorders? A comprehensive systematic review and meta-analysis. Eur J Nutr. 2016;55(3):897-906.

5. Gibson PR. History of the low FODMAP diet. J Gastroenterol Hepatol. 2017;32:5-7. 6. Tuck CJ, Muir JG, Barrett JS, Gibson PR. Fermentable oligosaccharides, disaccharides, monosaccharides and polyols: Role in irritable bowel syndrome. Expert Rev Gastroenterol Hepatol. 2014;8(7):819-34.

7. Halmos EP, Gibson PR. Controversies and reality of the FODMAP diet for patients with irritable bowel syndrome. J Gastroenterol Hepatol. 2019;34(7):1134-42.

8. Mitchell H, Porter J, Gibson PR, Barrett J, Garg M. Review article: implementation of a diet low in FODMAPs for patients with irritable bowel syndrome-directions for future research. Aliment Pharmacol Ther. 2019;49(2):124-39.

9. Dolan R, Chey WD, Eswaran S. The role of diet in the management of irritable bowel syndrome: a focus on FODMAPs. Expert Rev Gastroenterol Hepatol. 2018;12(6):607-15. 10. Whelan K, Martin LD, Staudacher HM, Lomer MCE. The low FODMAP diet in the management of irritable bowel syndrome: an evidence-based review of FODMAP restriction, reintroduction and personalisation in clinical practice. J Hum Nutr Diet. 2018;31(2):239-55.

11. Barrett JS. How to institute the low-FODMAP diet. J Gastroenterol Hepatol. 2017;32:8-10

12. Hill P, Muir JG, Gibson PR. Controversies and Recent Developments of the Low FODMAP Diet Efficacy of the Low-FODMAP Diet in Patients With Irritable Bowe Syndrome. Gastroenterol Hepatol (N Y). 2017;13(1):36-45.

13. Ireton-Jones $\mathrm{C}$. The low FODMAP diet: Fundamental therapy in the management of irritable bowel syndrome. Curr Opin Clin Nutr Metab Care. 2017;20(5):414-9.

14. Bellini M, Tonarelli S, Nagy AG, Pancetti A, Costa F, Ricchiuti A, et al. Low FODMAP diet: Evidence, doubts, and hopes. Nutrients. 2020;12(1):1-21.

15. Ordem dos Nutricionistas. Profissão de nutricionista [Internet]. [cited 2020 May 25] Available from: https://www.ordemdosnutricionistas.pt/ver.php?cod=0AOD.

16. Dietetics I. Nutrition Care Process and Model Part I: The 2008 Update. J Am Diet Assoc. 2008;108(7):1113-7.

17. Rao SSC, Yu S, Fedewa A. Systematic review: Dietary fibre and FODMAPrestricted diet in the management of constipation and irritable bowel syndrome. Aliment Pharmacol Ther. 2015;41(12):1256-70.

18. Su H, Li YT, Heitkemper MM, Zia J. Effects of Low-FODMAPS Diet on Irritable Bowel Syndrome Symptoms and Gut Microbiome. Gastroenterol Nurs. 2019;42(2):150-8.

19. Vakil N. Dietary Fermentable Oligosaccharides, Disaccharides, Monosaccharides, and Polyols (FODMAPs) and Gastrointestinal Disease. Nutr Clin Pract. 2018;33(4):468-75. 20. Shepherd SJ, Lomer MCE, Gibson PR. Short-chain carbohydrates and functional gastrointestinal disorders. Am J Gastroenterol. 2013;108(5):707-17.

21. Monash University. Research at Monash University [Internet]. [cited 2020 May 1]. Available from: https://www.monashfodmap.com/about-fodmap-and-ibs/researchmonash-university/.

22. Ong DK, Mitchell SB, Barrett JS, Shepherd SJ, Irving PM, Biesiekierski JR, et al.
Manipulation of dietary short chain carbohydrates alters the pattern of gas production and genesis of symptoms in irritable bowel syndrome. J Gastroenterol Hepatol. 2010;25(8):1366-73.

23. Monash University. FODMAPs and Irritable Bowel Syndrome [Internet]. 2019 [cited 2020 May 6]. Available from: https://www.monashfodmap.com/about-fodmap-and-ibs/. 24. Varney J, Barrett J, Scarlata K, Catsos P, Gibson PR, Muir JG. FODMAPs: food composition, defining cutoff values and international application. J Gastroenterol Hepatol. 2017;32:53-61.

25. Tuck C, Barrett J. Re-challenging FODMAPs: the low FODMAP diet phase two. J Gastroenterol Hepatol. 2017;32:11-5.

26. Krogsgaard LR, Lyngesen M, Bytzer P. Systematic review: quality of trials on the symptomatic effects of the low FODMAP diet for irritable bowel syndrome. Aliment Pharmacol Ther. 2017;45(12):1506-13.

27. Halmos EP, Power VA, Shepherd SJ, Gibson PR, Muir JG. A diet low in FODMAPs reduces symptoms of irritable bowel syndrome. Gastroenterology. 2014;146(1):6775.e5.

28. Whigham L, Joyce T, Harper $G$ et al. Clinical effectiveness and economic costs of group versus one-to-one education for short-chain fermentable carbohydrate restriction (low FODMAP diet) in the management of irritable bowel syndrome. J Hum Nutr Diet. 2005;28(6):687-696.

29. Tuck CJ, Reed DE, Muir JG, Vanner SJ. Implementation of the low FODMAP diet in functional gastrointestinal symptoms: A real-world experience. Neurogastroenterol Motil. 2020;32(1):1-13.

30. Böhn L, Störsrud S, Liljebo T, Collin L, Lindfors P, Törnblom H, et al. Diet Low in FODMAPs Reduces Symptoms of Irritable Bowel Syndrome as Well as Traditional Dietary Advice: A Randomized Controlled Trial. Gastroenterology. 2015;149(6):13991407.e2.

31. O'Keeffe M, Jansen C, Martin L, Williams M, Seamark L, Staudacher HM, et al. Long-term impact of the low-FODMAP diet on gastrointestinal symptoms, dietary intake, patient acceptability, and healthcare utilization in irritable bowel syndrome. Neurogastroenterol Motil. 2018;30(1):1-13.

32. Monash University. Your complete on-the-go guide to the FODMAP Diet [Internet]. [cited 2020 May 7]. Available from: https://www.monashfodmap.com/ibs-central/ihave-ibs/get-the-app/.

33. Tuck C, Ly E, Bogatyrev A, Costetsou I, Gibson P, Barrett J, et al. Fermentable short chain carbohydrate (FODMAP) content of common plant-based foods and processed foods suitable for vegetarian- and vegan-based eating patterns. J Hum Nutr Diet. 2018;31(3):422-35.

34. Staudacher HM, Whelan K. Altered gastrointestinal microbiota in irritable bowel syndrome and its modification by diet: Probiotics, prebiotics and the low FODMAP diet. Proc Nutr Soc. 2016;75(3):306-18.

35. Slomski A. The Low-FODMAP Diet Helps IBS Symptoms, but Questions Remain. JAMA - J Am Med Assoc. 2020;323(11):1029-31.

36. Gibson PR. The evidence base for efficacy of the low FODMAP diet in irritable bowel syndrome: is it ready for prime time as a first-line therapy? J Gastroenterol Hepatol. 2017;32:32-5.

37. Ooi SL, Correa D, Pak SC. Probiotics, prebiotics, and low FODMAP diet for irritable bowel syndrome - What is the current evidence? Complement Ther Med. 2019;43(January):73-80. 